\title{
EXTRACCION DE TRABAJO Y CAMBIO TECNICO: EL CASO DE LA FABRICA MCCORMICK DE CHICAGO, 1880-1900
}

\author{
LUIS TOHARIA \\ Unlversidad de Alcalá de Henares
}

\section{Introducción}

Según la teoría económica dominante, la teoría neoclásica, la única razón que explica la introducción de nuevas técnicas en el proceso productivo es que éstas son más eficientes o productivas que las conocidas hasta la fecha. Las razones por las que aparecen esas nuevas técnicas pertenecen a dominios extraeconómicos, es decir, las variables económicas no se consideran un factor relevante en dicha aparición. Por otra parte, los escritos recientes de algunos autores pertenecientes a la línea de pensamiento marxista, de entre los que destaca Stephen Marglin (1974), parecen haber sostenido que la introducción de cambios técnicos, y más concretamente de la división del trabajo, responde a un deseo por parte de los capitalistas de controlar mejor a sus obreros más que a razones de eficiencia productiva. En este artículo partimos de un enfoque teórico que pone de relieve que la «eficiencia» y el «control» no son variables contradictorias, sino complementarias. Es decir, puede haber cambios técnicos que respondan a razones de eficiencia, que llamaremos «físicos", y cambios técnicos que correspondan a razones de control, que llamaremos «extractivos». Nuestra crítica a la teoría neoclásica no es, por tanto, que sea errónea, sino que sólo es parcialmente correcta. El objetivo de este trabajo es poner de relieve, mediante el estudio concreto de una serie de cambios técnicos ocurridos en una misma planta en un período relativamente corto de tiempo, que los cámbios extractivos tienen relevancia empírica. El caso analizado es el de la fábrica McCormick, de Chicago, en las dos últimas décadas del siglo xIX. Por supuesto, sería arriesgado generalizar estos resultados tan concretos. Por ello, esta investigación debe considerarse únicamente como un ejemplo en el que el marco teórico propuesto resulta validado y como una invitación a posteriores investigaciones, tal vez menos distantes en el tiempo $y$, sobre todo, en el espacio, que confirmen o rechacen sus hallazgos.

Es importante resaltar que el hecho de que los cambios extractivos tengan relevancia no es meramente un hallazgo académico, sino que tiene impor- 
tantes consecuencias para nuestra comprensión del llamado «progreso tecnológico». Si el enfoque adoptado en este artículo es correcto, implica que el progreso tecnológico no es un fenómeno necesariamente científico y neutral, sino que puede contener elementos políticos que lo condicionen. La idea del determinismo tecnológico es, indudablemente, una de las ideas predominantes en las sociedades actuales. Si de algo sirve este artículo, debería ser como una modesta aportación al derrumbamiento de dicha idea.

En el apartado 2 del artículo se presenta en detalle el enfoque teórico en el que se enmarca el trabajo. El apartado 3 proporciona la información básica necesaria acerca de la fábrica McCormick, entorno en el que tuvieron lugar los cambios técnicos, sucesivamente analizados en los apartados 4, 5 y 6 . Por último, en el apartado final se resumen las principales conclusiones del artículo.

\section{Marco teórico}

De acuerdo con la teoría neoclásica, el proceso productivo viene definido por el conjunto de posibilidades técnicas (o «tecnología») existente en un momento dado del tiempo en función del estado de los conocimientos científicos y de ingeniería, y que los economistas sintetizan en la función de producción. En este contexto, la elección de la técnica concreta que utilizará la empresa vendrá determinada, siguiendo el principio de la maximización de los beneficios, por el precio relativo de los factores de producción. Según este esquema, si la tecnología no varía, los cambios técnicos vendrán explicados por la alteración del precio relativo de los factores. Así, si aumenta el coste del trabajo relativo al del capital, a la empresa le resultará rentable sustituir la técnica que esté usando por otra más intensiva en capital, elegida entre las sintetizadas por la función de producción conocida. Estos cambios técnicos deben distinguirse conceptualmente de los "cambios tecnológicos», es decir, de los cambios que suponen una alteración del conjunto de la función de producción. Dichos cambios vienen definidos por una variación de la productividad de los factores o cantidad de producto por unidad de factor. Conforme con su forma de concebir el proceso productivo y la función de producción, la teoría neoclásica considera que los cambios tecnológicos vienen determinados por los avances de la ciencia y la ingeniería y, por tanto, son independientes de la evolución de las variables económicas. Dicho con otras palabras, la teoría neoclásica no proporciona ninguna explicación teórica de los cambios tecnológicos ${ }^{1}$.

- A este respecto, cabe señalar que es erróneo argumentar que los cambios tecnológicos pueden explícarse con referencia a los cambios de los precios relativos 
El enfoque adoptado en este artículo difiere sustancialmente del análisis neoclásico que acabamos de exponer sucintamente. Nuestra concepción del proceso productivo no es meramente técnico-económica, sino que contiene elementos sociales y políticos fundamentales, que podemos agrupar bajo la denominación de «relaciones laborales». o «relaciones industriales» ${ }^{2}$. Resultaría absurdo y contrario a toda observación empírica negar que dichas relaciones tienen importancia para el proceso productivo de la empresa, sobre todo en economías capitalistas caracterizadas por la existencia de una gran mayoría de personas que realizan su trabajo como asalariados, es decir, por cuenta ajena. $\mathrm{Y}$, sin embargo, en la teoría neoclásica de la empresa esas relaciones son irrelevantes, como tan acertadamente señaló ya hace tiempo Samuelson (1957). El obviar la clara deficiencia que ese supuesto implica, lo que permitirá establecer una conexión entre los cambios tecnológicos y las rela. ciones laborales, es lo que pretende conseguir el enfoque aquí adoptado.

Nuestro planteamiento teórico parte de una función de producción que relaciona las cantidades de factores (capital y trabajo) con la cantidad de producto:

$$
Y=F(K, L)
$$

Esta función es similar a la postulada por la teoría neoclásica, con la única salvedad de que uno de los factores, el trabajo, no puede ser adquirido directamente en el mercado, sino que debe ser «producido» o «extraído» por la propia empresa a partir de la capacidad o disponibilidad para trabajar, o fuerza de trabajo, que es lo que se adquiere en el mercado. Así, pues, el proceso productivo está constituido no solamente por elementos técnico-económicos (la conversión de trabajo y capital en producto de la forma más eficiente posible), sino también por elementos cuyo cariz es más social o político (la conversión de fuerza de trabajo en trabajo efectivo) ${ }^{3}$.

de los factores, como sostenía nada menos que John Hicks (véase Rosenberg, 1969; también Temin, 1966, 1971). Y también deben mencionarse los esfuerzos realizados por toda una serie de economistas para proporcionar explicaciones de dichos cambios (véase la síntesis de Nelson, 1981), si bien sus investigaciones permanecen al margen del cuerpo central de la teoría neoclásica y en muchos casos han seguido caminos heterodoxos que las han alejado de ella.

2 O el "labor problem" del que hablaba Frederick Winslow Taylor (1895).

3 Este enfoque está en la línea de los trabajos recientes de autores marxistas tales como Braverman (1974), Gintis (1976), Edwards (1979) y Vegara (1981). Entre las aplicaciones teóricas recientes de la distinción entre trabajo y fuerza de trabajo, cuyo origen se remonta a Marx, cabe destacar, en primer lugar, la de Gintis y Bowles (1981), quienes, partiendo de una función de producción similar a la propuesta antes, han intentado proporcionar una explicación del paro voluntario keynesiano. Por otra parte, Reich y Devine (1981) la han utilizado para analizar la cuestión de la eficiencia, entendida como bienestar social, de la división del 
La forma más sencilla de expresar la relación existente entre trabajo $(L)$ y fuerza de trabajo $(P)$ sería postular la existencia de un factor de conversión o extracción $(e)$ entre ellos:

$$
L=e \cdot P
$$

El problema es analizar los factores que determinan el coeficiente $e$. Implícitamente, la teoría neoclásica supone que dichos factores son exógenos al proceso productivo, lo que equivale a decir que, en un momento dado del tiempo, $e$ es constante y que sus posibles variaciones en el tiempo no son atribuibles, en particular, a factores relacionados con lo que hemos llamado "relaciones laborales». En nuestro enfoque, $e$ no es una constante, sino una función; es decir, el problema de la extracción no tiene una solución automática, sino que debe ser resuelto a diario por las empresas. Es evidente que éstas tratarán de poner en pie unos mecanismos institucionales que faciliten la conversión, es decir, que maximicen el coeficiente $e$. Esos mecanismos constituyen lo que Richard Edwards ha llamado «sistema de control», el cual, según dicho autor, contiene tres elementos fundamentales: la dirección de las tareas a realizar, la supervisión y evaluación del trabajo realizado y la disciplina (Edwards, 1979, p. 18). Si el empresario pudiera controlar todas y cada una de las acciones y movimientos de sus empleados, sin que ello le supusiera coste alguno, y si el hacerlo le garantizara un rendimiento máximo de sus empleados, es evidente que lo haría. Pero, por una parte, le resultaría costoso, entre otras cosas por el personal de supervisión que necesitaría ${ }^{4}, y$, por otra, no tendría garantías de que los empleados no opondrían resistencia, individual o colectivamente, si consideraran la supervisión excesiva, lo cual redundaría en una disminución de la eficacia de la conversión de fuerza de trabajo en trabajo. En suma, el empresario se enfrenta a un problema de optimización en la extracción de trabajo cuya existencia le proporciona un incentivo para introducir cambios en el sistema de control empleado cuando las condiciones lo hagan aconsejable y posible. Así, por ejemplo, como han señalado e investigado Edwards (1979) y Gordon, Edwards y Reich (1982), la tendencia histórica observada en Estados Unidos ha sido la sustitución de un sistema de control «simple», en el cual el empresario era el encargado directo de la extracción, por sistemas estructuráles en los cuales los mecanismos de extracción quedan insertos en las estructuras técnicas y organizativas de las empresas.

Los comentarios anteriores sugieren que la extracción de trabajo tiene

trabajo elegida por una sociedad capitalista en comparación con la que existiría en un sistema de cooperativas obreras.

4 Al que, además, habría que supervisar de alguna manera. 
un elemento de continuidad temporal fundamental, es decir, que no basta con que la extracción se produzca en un momento aislado del tiempo, sino que el sistema de control existente tiene que tratar de garantizar que dicha extracción podrá tener lugar en el futuro. Dicho con otras palabras, el sistema de control tiene no sólo que extraer trabajo, sino también mantener o reproducir las condiciones que permiten que dicha extracción tenga lugar. Por ejemplo, un sistema que sea particularmente penoso para los empleados es probable que no tenga mucha viabilidad a largo plazo debido a la fuerte resistencia que generaría, a menos, desde luego, que existan estructuras sociales más amplias que el ámbito de la empresa que respalden dicho sistema $y$, de hecho, que lo impongan. La dimensión temporal que estamos destacando implica que si se plantea un conflicto entre la extracción de trabajo y la reproducción del sistema de extracción, esta última será prioritaria. Así, todo sistema que, aunque produzca trabajo, no garantice o amenace potencialmente su reproducción será un sistema que el empresario tratará de cambiar tarde o temprano ${ }^{5}$. De las amenazas potenciales a la reproducción cabe destacar la posibilidad de que el proceso productivo esté controlado, en sus aspectos fundamentales, por el colectivo de trabajadores o por grupos más reducidos que ocupan un puesto clave dentro de la cadena productiva. En este sentido, la dimensión temporal que estamos destacando proporciona una posible explicación del interés, observado históricamente, de los capitalistas por ser ellos los gestores del proceso productivo.

Nuestro análisis de la dimensión temporal del problema de la extracción ha puesto de relieve otra de las características fundamentales del mismo. La viabilidad del sistema de control requiere que sea aceptado por los trabajadores. Obviamente, en esta aceptación entrarán factores cuyo ámbito es superior a la empresa. Por ejemplo, la historia obrera está llena de casos de actuaciones represoras del Estado para imponer a los trabajadores condiciones de trabajo ante las que se rebelaban. Lo que estamos diciendo, en definitiva, es que, en una sociedad capitalista, la empresa no puede ser considerada de manera aislada, $y$, en concreto, no puede dejarse de lado el importante papel que puede desempeñar el Estado en conseguir que se acepte el sistema de control vigente $y$, por tanto, en garantizar su reproducción.

Una vez presentado el enfoque teórico del proceso productivo adoptado en este artículo, podemos aplicarlo al problema que nos interesa: el cambio tecnológico. ¿Por qué introducen las empresas capitalistas cambios tecnológicos? Según la teoría neoclásica, la razón es que la nueva técnica es más eficiente, es decir, su productividad por unidad de factor utilizada es mayor, ya que, si no fuera así, la rentabilidad de la empresa se vería en peligro y se

${ }^{3}$ Subrayo la palabra tratará para hacer hincapié en que esto no siempre será posible $\mathrm{y}$, no digamos, automático. 
trataría de una decisión suicida. El enfoque propuesto en los párrafos anteriores proporciona una respuesta algo diferente y, pienso, más completa. Desde luego, las nuevas técnicas son introducidas porque son más eficientes, pero el término eficiencia o productividad tiene, en nuestro enfoque, una doble vertiente: una relativa al proceso técnico-económico, similar a la identificada por la teoría neoclásica, que llamaremos "física», y otra relativa al proceso de extracción de trabajo, que llamaremos «extractiva». Esta segunda posibilidad es la que pone de relieve nuestro enfoque, ya que, en lo que se refiere a la primera, no aporta elementos nuevos con respecto a la teoría neoclásica. Lo que estamos argumentando es que es posible endogeneizar en parte los cambios tecnológicos, en concreto aquellos cuya motivación pueda estar relacionada con los problemas de extracción de trabajo. $Y$ en este artículo, recurriendo a un análisis histórico de un caso concreto, pretendemos sostener que esta distinción tiene relevancia empírica.

Una primera consecuencia que se deduce del análisis anterior es que la «eficiencia» y el "control" no son dos dimensiones contradictorias, como parece deducirse de los trabajos pioneros de Stephen Marglin (1974), sobre el origen de la división del trabajo en las sociedades capitalistas, y de Katherine Stone (1974), sobre la introducción de estructuras de empleo en la industria del acero de Estados Unidos. Más bien se trata de dos tipos diferentes de eficiencia, una física y otra extractiva, que pueden o no estar en conflicto. Ahora bien, lo que nuestro enfoque sugiere es que, debido a su proyección temporal de reproducción del sistema de control, la dimensión extractiva tendrá prioridad sobre la física, por lo que los estudios de Marglin y Stone encajan perfectamente dentro del esquema que hemos propuesto.

La pregunta que surge a continuación es hasta qué punto son separables los dos tipos de cambios tecnológicos identificados en los párrafos anteriores. Nuestro interés se centra en las motivaciones para introducir cambios técnicos. A ese nivel, está claro que las motivaciones pueden ser de uno u otro tipo o de ambos a la vez, y siempre recordando la dimensión temporal del problema de la extracción. Por ejemplo, la sustitución de una máquina de escribir manual por una eléctrica parece un caso claro de cambio tecnológico «físico», ya que si el que la maneja no quiere, no consigue un mayor rendimiento del trabajador. Por otra parte, un cambio en la estructuración física del centro de trabajo que facilite la supervisión por parte del empresario es claramente un cambio de tipo «extractivo». Por último, los nuevos sistemas de «procesamiento de textos» con ordenadores no sólo suponen una mejora física, sino que, además, permiten al propio ordenador supervisar el rendimiento del trabajador. A posteriori, sólo sabemos que ambos tipos de mejora se han producido, pero no sabemos si la motivación fue "física» o «extractiva». Para averiguarlo sería necesario analizar en detalle la introducción de la técnica en 
cuestión. El problema de separar las motivaciones físicas y las extractivas constituye, sin duda, el reto empírico más importante para nuestro enfoque, ya que siempre se puede aducir que las motivaciones fueron únicamente físicas, y por tanto políticamente neutrales, y lo que pasó fue que tuvo consecuencias extractivas imprevistas y a priori indeseadas. En todo caso, lo que nuestro enfoque sugiere es que ante dos posibles nuevas técnicas, igualmente eficientes desde el punto de vista físico, en una sociedad capitalista será elegida aquella que produzca una extracción más eficiente o, en todo caso, aquella que garantice mejor la reproducción del sistema de control.

Los casos que presentamos a continuación ponen de relieve que las motivaciones extractivas fueron determinantes en la introducción de los cambios tecnológicos analizados. Ello no quiere decir que todos los cambios tecnológicos tengan siempre y en todo lugar motivaciones extractivas, pero sí quiere decir que no responden necesaria y únicamente a motivaciones «físicas»; en definitiva, que la tecnología no es, al menos no en todos los casos, un proceso que se imponga del exterior como algo neutral y determinista, sino que puede ser la respuesta a problemas internos a la empresa, especialmente a problemas referentes a las relaciones laborales.

\section{La fábrica McCormick, 1847-1902}

El objetivo de este apartado es presentar la información básica referente a la fábrica McCormick que sirva como marco de referencia para analizar los cambios técnicos que constituyen el interés principal del artículo.

Cyrus Hall McCormick, un joven herrero de Virginia ${ }^{6}$, patentó su primera segadora mecánica en $1834^{7}$, si bien hasta 1847 no puede decirse que empezara a producirlas industrialmente, con la ayuda de sus hermanos, en Chicago $^{8}$. Hasta 1880 , la empresa McCormick fue propiedad de los hermanos, principalmente de Cyrus. En 1880, la empresa se convirtió en sociedad

- La mejor biografia de Cyrus Hall McCormick es la de Hutchinson (1930, 1935). Mi relato de la compañía McCormick se basa en esta biografía, así como en Ozanne (1967, 1968), Hounshell (1978) y en datos primarios recogidos en la McCormick Collection, que se halla en los Archivos de la State Historical Society of Wisconsin, en Madison (Wis., Estados Unidos). Esta increíble colección ha servido de base para la investigación (Toharia, 1979) en la que se cimenta este artículo.

- Aunque Cyrus McCormick no fue el primero en patentar una segadora mecánica, puesto que Obed Hussey patentó la suya un año antes, parece que fue el primero en fabricar una que funcionara satisfactoriamente en 1831. Los detalles del debate sobre quién fue el verdadero inventor pueden hallarse en Hutchinson (1930).

- El artículo clásico entre el desfase existente entre la invención de la cosechadora mecánica y su aplicación agrícola a gran escala es el de Paul David (1975). Véanse también las críticas a este trabajo de Olmstead (1975) y Jones (1977). 
anónima, si bien la familia retuvo una participación mayoritaria en la misma. En 1902, la compañía McCormick y sus principales competidores se fusionaron para crear la International Harvester Company, empresa que, al nacer, ocupaba una posición casi monopolista en la industria de fabricación de maquinaria agrícola y que hoy en día es una potente multinacional, a pesar de sus recientes problemas financieros, en la que la familia McCormick sigue desempeñando un papel de relieve. La compañía McCormick fue, pues, una empresa pionera en el desarrollo económico americano, tanto por su importancia en el florecimiento industrial de Chicago ${ }^{9}$ como por el enorme avance de la productividad agrícola que produjo la introducción de la segadora y, más tarde, la cosechadora mecánicas (véase Parker y Klein, 1966).

A lo largo de toda su historia, la compañía McCormick se especializó en la producción de maquinaria agrícola, que llevó a cabo en una única fábrica, cuyo emplazamiento cambió a raíz del incendio de octubre de 1871 , el cual, al destruir prácticamente la ciudad de Chicago, arrasó la vieja fábrica en la que los McCormick producían desde 1847. La nueva fábrica, cuya construcción fue posible gracias a la determinación y la fortuna de Cyrus McCormick, fue edificada en unos terrenos que permitieran su expansión posterior y, así, la fábrica permaneció en la misma ubicación hasta su demolición final en 1962.

En la fabricación de las segadoras se utilizaban dos tipos de materiales: la madera y el hierro. Hasta 1880 , el proceso productivo era bastante artesanal y la división del trabajo rudimentaria, aunque no del todo inexistente. El peso de la producción recaía en carpinteros, herreros y moldeadores cualificados, a los que había que añadir los pintores, que daban a las máquinas su aspecto final. Todos ellos eran ayudados por una abundante mano de obra no cualificada, como lo demuestra el hecho de que cerca del 60 por 100 de los trabajadores de producción percibía el salario de peón o menos ${ }^{10}$. Es en este sentido en el que cabe decir que la división del trabajo era rudimentaria, ya que si bien los trabajadores cualificados eran ayudados en las tareas más elementales por los no cualificados, éstos no estaban especializados. A finales de la década de 1860 parece que se produjo una cierta tendencia hacia la especialización de los obreros, que fue posible gracias a algunas máquinas importadas de las armerías del Este por Leander McCormick, hermano menor de Cyrus y superintendente de la fábrica ". El aumento del porcentaje de trabajadores pagados por piezas (del 16 por 100 en abril de 1867 al 30 por

\footnotetext{
- Por ejemplo, el catálogo de la compañia para el año 1880 afirmaba que era una de las tábricas más grandes del mundo y el de 1885 presentaba un grabado de la fábrica cuyo pie rezaba: "la mayor del mundo".

${ }^{10}$ Estos datos proceden del análisis de las nóminas de la compañia, que se conservan en la McCormick Collection.

" Véase, a este respecto, Hounshell, 1978, p. 240.
} 
100 en abril de 1871) así parece atestiguarlo. El incendio de 1871 detuvo este proceso, que no volvería a reanudarse, como veremos, hasta 1880 .

La fundición merece un comentario aparte. Incorporada a la fábrica en 1854, parece que siempre se mantuvo un tanto al margen de ella. Así, los moldeadores eran pagados por piezas, práctica apenas vigente en el resto de la factoría. Por otra parte, en las nóminas de la compañía, la fundición siempre figuraba separada de la —así llamada - «fábrica». Por último, los moldeadores cualificados pertenecían en su mayoría al poderoso Sindicato de Moldeadores, el cual, como le escribia Leander a Cyrus en 1867, «controla completamente nuestro taller» ${ }^{12}$. Cabría añadir otro factor más, $y$ es que los McCormick, dada su formación de herreros, no entendían o no estaban interesados en entender el proceso de vaciado; les bastaba con que el taller de fundición proporcionara las piezas necesarias para la fabricación de las máquinas. Todo ello implica que existia una dualidad entre la «fábrica» y la fundición y que esta última llegó a convertirse en el punto clave del proceso productivo, lo que permitió a los moldeadores ponerse en huelga y conseguir mejoras salariales en diversas ocasiones ${ }^{13}$. Un último punto que merece la pena señalar, en lo que se refiere a la fundición, es que los moldeadores ejecutaban ellos mismos la práctica totalidad de las tareas del proceso de vaciado, con la asistencia de, a lo sumo, un ayudante, como estipulaba una de las reglas más importantes del sindicako. Por tanto, mientras que en el resto de la fábrica se produjo, como vimos antes, una cierta tendencia hacia la especialización de los obreros, el taller de fundición siguió utilizando, al menos hasta 1885 , como veremos más adelante, la misma técnica artesanal de producción.

Las dos últimas décadas del siglo fueron fundamentales desde el punto de vista de la expansión de la empresa. En ellos, la compañía pasó de ser un floreciente negocio familiar a ser una gran empresa industrial: la producción y los beneficios se dispararon, la gama de maquinaria agrícola producida y el empleo se multiplicó por diez. Fue en esta última parte del siglo cuando tuvieron lugar los cambios técnicos que constituyen el objeto de este artículo, y que pasamos a analizar a continuación.

\section{La introducción en 1880 de las técnicas utilizadas en las armerias}

Como vimos antes, el incendio de 1871 destruyó completamente la vieja fábrica de los McCormick. Pero, al parecer, supuso algo más: la pérdida de

12 Citado en Ozanne, 1967, p. 7.

13 Como señala Ozanne (1967), estas mejoras beneficiaban en muchas ocasiones no sólo a los moldeadores, sino también a los demás obreros de la tábrica. 
interés por parte de Leander. Las tensiones entre los dos hermanos eran antiguas ${ }^{14}, y$ procedían de que la percepción de Cyrus de que el potencial de mercado era cada vez mayor chocaba con los constantes problemas a los que Leander tenía que hacer frente para sacar adelante la producción ${ }^{15}$, que le llevaba a una postura mucho menos expansionista. De hecho, algunos años antes del incendio, Cyrus ya había propuesto a su hermano trasladar la fábrica de emplazamiento para permitir su expansión, a lo que éste se había opuesto. Así, durante los primeros años de funcionamiento de la nueva fábrica, a los problemas inherentes a su puesta en marcha se unieron no sólo los problemas económicos generales de la «Gran Depresión» de la década de 1870, sino también el malestar de Leander, superintendente de la fábrica y, por tanto, máximo responsable directo de la producción. Por ejemplo, Hounshell aduce la insatisfacción de Leander como una de las principales razones que explican la cifra de producción correspondiente a 1877 , la más baja de toda la década ${ }^{16}$.

Al parecer, Cyrus tampoco estaba muy satisfecho con la gestión de su hermano al frente de la fábrica. Había contratado a un experto en patentes, Charles Colahan, que también era un mecánico experto, y decidió enviarle a visitar la factoría. En una carta a Cyrus, Colahan le manifestaba la siguiente opinión: «debe instaurar más sistema, orden y disciplina [en la fábrica] para garantizar una construcción perfecta previa a su envío» ${ }^{17}$. Como sugiere Hounshell, al parecer, Leander había descuidado totalmente su tarea de director de la producción.

La consecuencia fue que, en mayo de 1880, el Consejo de Administración de la compañía decidió despedir a Leander y nombrar un nuevo superintendente, Lewis Wilkinson, un mecánico de Nueva Inglaterra que tenía experiencia en la fabricación de armas y de máquinas de coser. Asimismo, Cyrus H. McCormick, Jr., hijo de Cyrus, fue nombrado su ayudante. La breve permanencia de Wilkinson en la empresa ${ }^{18}$ fue decisiva para la organización del proceso productivo que iba a ser adoptada a partir de entonces, así como fundamental para permitir el gran aumento de la producción expe-

14 Asi queda reflejado en la biografia de Hutchinson (1935).

is Leander era el encargado de la fábrica mientras que Cyrus se ocupaba fundamentalmente de la consecución y renovación de sus patentes. Asimismo, Cyrus era el que determinaba los objetivos de producción de la empresa, así como los cambios que había que introducir en los modelos fabricados, todo ello en función de su apreciación personal de la situación del mercado.

${ }_{16}$ Citado en Hounshell, 1978, p. 256. Durante toda la década, las cifras de producción y venta se mantuvieron en niveles muy bajos. Las ventas fueron especialmente malas en 1876 debido a la crisis económica, lo que también explica en parte la baja producción de 1877 .

${ }_{17}$ Citado en Hounshell, 1978, pp. 254-255.

" Sólo un año. Se desconocen las razones por las que no permaneció en la empresa más tiempo. 
rimentado por la empresa a principios de la década de 1880 . Lo primero que hizo Wilkinson, como señala Hounshell ${ }^{19}$, fue imponer orden y disciplina en la fábrica, algo que Leander llevaba algunos años sin hacer, es decir, corregir los defectos apuntados por Colahan unos años antes.

Sin embargo, la contribución más importante de Wilkinson fue la puesta en práctica de una nueva concepción del proceso productivo, caracterizada por los tres aspectos siguientes:

a) Desde el punto de vista de la producción, aumentó el grado de estandardización del producto. Se adoptó por primera vez un juego completo de plantillas y calibradores y se construyó una «máquina-patrón», es decir, una máquina que debía servir de modelo para la fabricación de las demás ${ }^{20}$. Esto parece indicar que el año que pasó Wilkinson en McCormick lo dedicó a remodelar la fábrica siguiendo el modelo de las armerías, dotándola de las herramientas especiales que eran características de dicho sistema, y cuya construcción, como ha señalado Merritt Roe Smith (1977), era uno de los elementos más importantes y costosos del sistema. Debe señalarse que todos estos cambios no afectaron al taller de fundición, que siguió funcionando igual que lo venía haciendo hasta entonces.

b) Desde el punto de vista de la organización de la producción, se creó el Departamento de Herramientas (Tool Room) ${ }^{21}$. Sus funciones eran diseñar y fabricar la maquinaria especial necesaria para la producción. Aunque la mayor estandardización del producto hizo necesaria la implantación de este departamento, ésta también representaba un intento de centralizar las tareas de entender y mejorar el proceso productivo en una pequeña célula alejada de los trabajadores directamente encargados de la producción. En suma, permitió a los directivos de la empresa adquirir un control mucho mayor de las diferentes tareas que conformaban el proceso productivo (a excepción del taller de fundición, como ya se ha señalado).

c) Por último, desde el punto de vista de los trabajadores, el cambio más significativo fue el importante aumento del porcentaje de obreros de la «fábrica» ${ }^{2}$ pagados por piezas en vez de por días. Dicho porcentaje pasó de 15,5 por 100 en 1880 a 32,6 por 100 en 1881 y 42 por 100 en $1882^{23}$.

19 Hounshell, 1978, p. 262.

20 Esto sucedió a mediados de la campaña productiva de 1881, como aparece reflejado en el Diario de Cyrus, Jr., McCormick Collection.

${ }_{21}$ Esto es lo que sugieren las declaraciones de testigos presenciales recogidas en "Manuscript History of the McCormick Works", de R. O. Johnson, trabajo preparado en 1931 y que se encuentra en la McCormick Collection. Sin embargo, no pude hallar ninguna evidencia directa sobre esta cuestión.

22 Es decir, excluida la fundición.

${ }^{23}$ Este porcentaje aumentaría posteriormente más despacio, pero de forma continua, alcanzando la cifra de alrededor del 60 por 100 hacia finales del siglo. 
La introducción a gran escala del trabajo por piezas o a destajo fue posible gracias a los cambios señalados en lo que se refiere a la mayor estandardización del producto. En efecto, la implantación de plantillas y calibradores implica que resulta más fácil identificar las tareas $\mathrm{y}$, por tanto, ponerles precio. Sin embargo, no hace obligatorio el trabajo a destajo, es decir, el imponer el pago por piezas constituye un elemento con entidad propia. Hay dos aspectos del trabajo a destajo que deben destacarse: primero, crea un fuerte incentivo a trabajar más intensamente y durante más tiempo, especialmente cuando, como en el caso de McCormick, permite obtener unos ingresos sustancialmente más elevados ${ }^{24}$; en segundo lugar, el trabajo a destajo reduce considerablemente la tarea de supervisión por parte del empresario, si bien el control conseguido es incompleto, porque sólo afecta al contenido de las tareas, pero no al ritmo de ejecución de las mismas ${ }^{25}$.

En suma, no cabe duda de que el año 1880 supuso un cambio decisivo de rumbo en lo que se refiere a la forma de organizar el proceso productivo en la fábrica McCormick. La pregunta que se plantea, a la luz del marco teórico esbozado en el apartado 2, es: ¿estuvieron motivados estos cambios por consideraciones «físicas» o "extractivas»? La evidencia presentada parece sugerir que nos encontramos ante un caso en el que la separación no es posible. Por una parte, parece claro que los problemas iniciales que dieron lugar a los cambios eran la falta de orden y disciplina en la fábrica, motivada por la dejadez de Leander. Es decir, el problema inicial parecía ser la total ineficiencia, por falta de interés o por la razón que fuera, de Leander para extraer trabajo de la fuerza de trabajo de que disponía. En este sentido, la motivación parece claramente extractiva. Sin embargo, para restablecer la eficiencia extractiva no era necesario introducir en toda su complejidad el sistema de producción de las armerías. Como hemos visto antes, la introducción de un sistema completo de plantillas y calibradores para conseguir un producto más homogéneo puede considerarse como un cambio "físico". Lo que ocurre es que el sistema de producción estandardizada de las armerías incluía también otros dos elementos: el trabajo a destajo y la creación del Departamento

${ }^{24}$ Un análisis de los trabajadores que cobraban por dias en 1880 y por piezas en 1881, basado en las nóminas de la empresa, revela que en el segundo año ganaban en promedio un 83 por 100 más que el año anterior. Los datos se refieren a 121 trabajadores, equivalentes a un 25 por 100 aproximadamente de la plantilla de la empresa.

${ }^{25}$ Para que el ritmo de ejecución sea elevado, el incentivo salarial de cobrar por piezas debe mantenerse elevado ya que si no el sistema se vicia y se deteriora. Hacia finales de siglo, las diferencias salariales entre los trabajadores pagados por piezas y los pagados por días se habian reducido considerablemente. Este problema del ritmo de ejecución era precisamente una de las críticas que Frederick Winslow Taylor hacía de los sistemas de pago por piezas simples. Véase, por ejemplo, Taylor (1895). 
de Herramientas. El primero es claramente un elemento extractivo, mientras que el segundo lo es solamente en la medida en que sus funciones no se limitan a la fabricación y reposición de plantillas, calibradores, troqueles, etc., sino que abarcan también el diseño del proceso productivo en todos sus niveles, como ocurrió en el caso de McCormick.

La conclusión que nos interesa destacar es que tanto la motivación inicial como algunos de los elementos fundamentales del nuevo sistema estaban relacionados con el problema de la conversión de fuerza de trabajo en trabajo efectivo.

\section{La mecanización del taller de fundición, $1885-86$}

Como ya hemos señalado anteriormente, el taller de fundición no se vio afectado por los importantes cambios organizativos introducidos en 1880 y siguió utilizando los mismos métodos artesanales que venía empleando desde hacía treinta años. Para entender mejor el cambio técnico que vamos a analizar en este apartado, es necesario describir brevemente las tareas ejecutadas por los moldeadores en sus operaciones de vaciado de las piezas de hierro macizo que llevaban los distintos modelos de cosechadoras ${ }^{26}$.

El vaciado consiste en rellenar con metal fundido un molde hueco hecho con una arcilla especial a partir de un patrón de madera o de hierro. $\mathrm{Al}$ moldeador se le entrega el patrón en dos mitades simétricas, lo que le permite fabricar el molde en otras dos mitades. Cada una de éstas la hace apisonando la arcilla alrededor del patrón en un recipiente especial. Cuando la arcilla ha sido debidamente apisonada se retira el patrón, se arreglan los posibles desperfectos producidos por el molde al retirar el patrón y se procede a fabricar la otra mitad del molde. Cuando las dos mitades ya están fabricadas se unen poniendo una encima de la otra, de tal forma que el recipiente especial, cuyo aspecto exterior es el de un cajón de madera, contiene en su interior el molde hueco cuya forma corresponde a la pieza que se quiere fabricar. A continuación se procede a verter el metal fundido a través del canal especial construido a tal efecto. Cuando el metal se ha enfriado se abren los recipientes, se limpian de arcilla las piezas fabricadas, se liman los posibles excesos de metal procedentes del relleno del canal de vertido y de los agujeros especiales hechos en la arcilla para permitir la salida del aire del molde cuando se vierte el metal, y se envía la pieza al departamento de acabado.

De las tareas que acabamos de describir en el proceso de vaciado hay dos que destacan en lo que se refiere a la destreza que requieren del mol-

${ }^{26}$ La descripción siguiente se basa en U.S. Employment Service (1938), así como en Roe y Lytle (1935). 
deador: el apisonamiento de la arcilla para darle la consistencia adecuada y la retirada del patrón y acabado final del molde. De todas formas, en el taller de fundición de McCormick, como ya hemos señalado, los moldeadores realizaban todas las tareas ellos mismos, con la única asistencia de un ayudante y con la posible excepción de la preparación de la arcilla especial y del hierro fundido, que probablemente estaban a cargo del capataz del taller con un grupo de peones.

Este sistema, por muy artesanal que fuera, nunca planteó problemas de estrangulamientos de la producción, y el taller de fundición respondió sin grandes problemas al enorme aumento de la producción experimentado en los primeros años de la década de 1880 , cuando la producción se duplicó con creces. Los problemas que surgieron fueron de otro tipo.

A la muerte de su padre, Cyrus $\mathrm{H}$. McCormick, Jr., fue nombrado presidente de la compañía, en 1884 . Tras un año en que la empresa había batido sus récords en cuanto a producción y beneficios, el joven Cyrus, Jr., decidió, en diciembre de ese año, reducir los salarios pagados a los obreros de su fábrica, por razones que no le han sobrevivido ${ }^{27}$. La reducción fue de un 10 por 100 para los trabajadores pagados por días y de un 15 por 100 para los pagados por piezas. Entre estos últimos se encontraban los moldeadores, que no tardaron mucho en replicar a la reducción salarial con una huelga, de la que salieron victoriosos, viéndose McCormick obligado a restablecer los niveles salariales anteriores. Como consecuencia de todo ello, el resultado final de la temporada productiva fue un descenso de la producción de un 11 por 100 y una reducción de los beneficios a la mitad de los obtenidos el año anterior.

La fuerza de los moldeadores y su sindicato quedó patente, una vez más, en la huelga de 1885 . No era, desde luego, la primera vez que sucedía esto y, seguramente, no le pillaba de sorpresa a Cyrus, Jr., quien en 1881 había anotado en su Diario que la compañía había advertido a los moldeadores que tuvieran cuidado con el curso suicida que estaban siguiendo ${ }^{28}$. En todo caso, parece claro que la empresa comprendía perfectamente que la fuerza de los moldeadores residía en el puesto clave que, como vimos antes, habían llegado

${ }^{27}$ Los rumores que corrieron entre los obreros de la fábrica indicaban que la reducción tenía la finalidad de financiar una donación de $\mathbf{4 0 0 . 0 0 0}$ dólares al McCormick Theological Seminary, fundado por Cyrus McCormick Sr. Sin embargo, la correspondencia entre Cyrus $\mathrm{Jr}$. y su madre a propósito de la huelga que siguió a la reducción no parece confirmarlo. Parece claro que la reducción pretendía ser temporal, como lo sugiere el hecho de que los "salarios por día" no fueron alterados en los libros de nóminas, es decir, la paga total que figuraba era igual al salario por día multiplicado por el número de días trabajados menos el 10 por 100 (los precios de las piezas no aparecían en las nóminas).

21 Según se refleja en el Diario de Cyrus Jr. El contenido de la amenaza implícita es una incógnita. 
a ocupar dentro del proceso productivo. De ahí que sus esfuerzos se centraran precisamente en alterar dicha posición.

En marzo de 1885 , es decir, poco después de la huelga de los moldeadores, la compañía McCormick había oído hablar de una máquina moldeadora fabricada por la Pneumatic Co., de Indianápolis. La carta enviada por McCormick pidiendo información sobre las máquinas resulta reveladora:

«Les agradeceremos nos envíen toda la información relativa a estos Moldes, su coste y forma de suministrar el aire comprimido. ¿Requieren hombres experimentados para hacerlos funcionar? ¿Cuánto tiempo haría falta para montar y poner en marcha un taller? Tenemos actualmente problemas con algunos de nuestros moldeadores y desearíamos recibir explicaciones detalladas y toda la información posible con respecto a sus máquinas...» (el subrayado es mío) ${ }^{29}$.

Un mes más tarde, los directivos de la compañía habían visto las máquinas y acordado comprarlas para la campaña productiva de 1886 , que empezaba, como siempre, en septiembre del año anterior. Entre septiembre de 1885 y marzo de 1886, la compañía gastó unos 70.000 dólares en «Máquinas de moldear para la fundición» ${ }^{30}$, lo que representaba el gasto más fuerte en maquinaria realizado por la empresa desde $1879 \mathrm{y}$, por tanto, probablemente de toda su historia. Armada con las máquinas moldeadoras, la compañía esperaba llevar a cabo la producción sin necesidad de recurrir a los moldeadores cualificados que la habían realizado hasta entonces.

¿Cuál fue la reacción de los moldeadores? Simplemente, nunca pensaron que las máquinas funcionarían y las consideraron como una excusa de la compañía para enfrentarse al sindicato en otras cuestiones más fundamentales y deshacerse de él. La revista del sindicato, en su número de marzo de 1886, explicaba así los hechos:

«... Cuando la fábrica cerró la temporada productiva en agosto del año pasado, era evidente que la empresa estaba dispuesta a deshacerse de los moldeadores, ya que en una convención de los representantes de las empresas de fabricación de maquinaria agrícola de Estados Unidos y Canadá celebrada en Indianápolis, aproximadamente el 15 de agosto de 1885 , con el fin de poner a

${ }^{29}$ Letterpress Book Domestic, núm. 268, p. 859. McCormick Collection.

30 Esta cifra procede de los informes financieros mensuales del tesorero de la compañía al Consejo de Administración. Esta cantidad es, por cierto, muy inferior al medio millón de dólares dado por Ozanne (1967). La fuente de Ozanne era la "Manuscript History", de R. O. Johnson. 
prueba una máquina de moldear que estaba en funcionamiento en la factoría Atlas de dicha ciudad..., la opinión unánime de todos estos representantes fue que la máquina nunca sería un éxito como máquina de moldear, con la única excepción del superintendente y capataz de la factoría McCormick. Ahora bien, hermanos, recordemos que estos hombres son ambos moldeadores... y que debían de saber que estas máquinas no funcionarían. ¿Cuál era su propósito al sustituir a los moldeadores por máquinas? El principal era que no hubiera hombres sindicados en ningún departamento de la fábrica... Cuando las máquinas fueron declaradas un fracaso... procedieron a introducir el sistema buck, llamando a aquellos que pensaban podian oponer menos resistencia a dicha petición... ${ }^{31}$.

La prohibición del sistema buck, consistente en que un moldeador pudiera tener más de un ayudante, lo que le permitía concentrarse en las tareas que requerían mayor destreza, era una de las reglas fundamentales del Sindicato de Moldeadores ${ }^{32}$. No es de extrañar, por tanto, que cuando los representantes del sindicato fueron a hablar con el presidente McCormick, en febrero de 1886, su protesta no se centró en la maquinaria introducida para sustituirles, sino en la violación de dicha norma y en el hecho de que la empresa hubiera empleado a cinco moldeadores no pertenecientes al sindicato. $\mathrm{La}$ respuesta del presidente fue contundente: por una parte, insistió en negociar a título individual con cada uno de sus empleados, sin obligar a los moldeadores «a trabajar con varios ayudantes a menos que fueran ellos los que así lo decidieran» ${ }^{33}$; por otra parte, declaró que la empresa emplearía a quien le pareciera adecuado, negándose a discriminar en función de la afiliación sindical. Mientras que un año antes las cuestiones en litigio habían girado en torno a los salarios, en esta ocasión se concedieron inmediatamente aumentos salariales, pero el problema del control de la fundición siguió siendo el importante ${ }^{34}$.

Tras esta respuesta de la empresa, los moldeadores, que durante ese año,

${ }^{31}$ Molders' Union Journal, marzo de 1886, consultado en la Biblioteca de la State Historical Society of Wisconsin.

${ }^{32}$ A este respecto, véase Ashworth (1915), p. 68, así como la historia del sindicato de Stockton (1821), especialmente pp. 179-185.

33 Esta cita procede del Diario de Cyrus Jr., que es la fuente de los acontecimientos narrados en este párrafo.

${ }^{34} \mathrm{La}$ información sobre las demandas y las conceslones salariales proceden de un recorte de prensa (The Morning News, 16 de febrero de 1886) conservado en la McCormick Collection. Esta cuestión no aparece mencionada en el Diario de Cyrus Jr., que sólo menciona el tema de la violación de la norma del sindicato: "Lste es uno de los grandes temas que preocupan al sindicato." 
de gran militancia sindical en Chicago, habían conseguido que la mayoría de los trabajadores de McCormick se afiliaran a los Knights of Labor, acordaron ir a la huelga, con el respaldo del resto de la factoría, lo que fue impedido por el cierre patronal de la fábrica. Los problemas de la fundición de McCormick coincidieron con la importante actividad sindical de esos meses en favor de la jornada de ocho horas. Los conocidos sucesos del $10^{\circ}$ de mayo de 1886 en Haymarket Square, en Chicago, a los que los moldeadores de McCormick no fueron ajenos, supusieron no sólo la derrota del movimiento en favor de la jornada de ocho horas, sino también la pérdida de toda posibilidad por parte de los moldeadores y su sindicato de recuperar la posición clave que habian ocupado antes en la fábrica McCormick ${ }^{35}$.

El caso de la mecanización del taller de fundición de la fábrica McCormick ha sido interpretado generalmente como un ejemplo clásico de una tecnología utilizada para eliminar a un sindicato poderoso ${ }^{36}$, similar a los muchos casos de oficios sustituidos por maquinaria existentes en la historia, en los cuales la resistencia de los obreros a perder su puesto de trabajo podía entenderse como una negativa a aceptar el progreso tecnológico como algo necesario y positivo ${ }^{77}$. En esos casos, parecería que se tratara de cambios tecnológicos cuya motivación era «física», aunque tuvieran evidentes consecuencias «extractivas», o, en todo caso, que la motivación fuera de ambos tipos. Sin embargo, la interpretación que se deduce de lo expuesto en las páginas anteriores con referencia al caso de la fundición de McCormick es diferente.

En primer lugar, está claro que la motivación para introducir las máquinas moldeadoras fue única y exclusivamente «extractiva», puesto que en ningún momento se planteó la empresa si el proceso de vaciado resultaría más barato o eficiente con las máquinas. La percepción inicial de los directivos de McCormick encajaba perfectamente con la famosa cita de Marx: «la introducción de maquinaria es la mejor forma de reprimir las huelgas” ${ }^{38}$. Sin embargo, el verdadero cambio introducido no fue la utilización de las máquinas. El funcionamiento de las mismas fue más que defectuoso y, en todo caso, no eran sustitutas de los moldeadores, puesto que sólo mecanizaban el apisonamiento de la arcilla. Esta era, sin duda, una de las tareas que requería mayor destreza dentro del proceso de vaciado, pero no lo suficiente como para poder ser representativa del proceso en su conjunto. El cambio fue, más bien, intro-

${ }^{35}$ Los hechos del $10^{\circ}$ de mayo de 1886 han sido relatados por muchos historiadores. Véase, por ejemplo, Brecher (1972), capítulo 2.

${ }_{36}$ Esta interpretación procede del trabajo de Ozanne (1967). Véanse Dubofsy (1975) y Henderson y Cohen (1979).

${ }_{37}$ Un caso contemporáneo al de los moldeadores es el de los trabajadores cualificados de la industria del acero; véanse Brody, 1960, caps. 2 y 3, y Stone, 1974,

especialmente pp. 63-67. I del Capital y aparece recogida en Henderson y Cohen
" La cita es del vol. I del (1979), 16 . 
ducir la posibilidad de que los moldeadores pudieran trabajar con más de un ayudante, concentrándose ellos en las tareas más delicadas y supervisando el trabajo de los ayudantes, encargados de las tareas más sencillas. Los hechos analizados demuestran que esto lo percibieron tanto Cyrus McCormick, Jr., presidente de la compañia, como el Sindicato de Moldeadores. La verdadera cuestión en litigio era el sistema de control que debía existir en la fundición. Con el sindicato en la fundición, la extracción de trabajo dependía de los moldeadores $\mathrm{y}$, aunque dicha extracción no fue problemática durante los primeros años de la década de 1880 , la huelga de 1885 puso de manifiesto que el sistema vigente no la garantizaba. Lo que realmente supuso la «mecanización" fue un cambio del sistema de control. A partir de entonces empezaron a regir las normas de la empresa en vez de las del sindicato. A la empresa no le importaba tanto el coste de instaurar un nuevo sistema de control como la amenaza potencial permanente que suponía la vigencia del antiguo, por muy eficiente que resultara desde el punto de vista productivo. Esto supone una ilustración del argumento presentado en el apartado teórico acerca de la importante dimensión temporal de la extracción de trabajo y de su preponderancia sobre la eficiencia física. Durante el año 1886, la empresa McCormick sacrificó una buena parte de su producción y sus beneficios en aras del cambio del sistema de extracción. $Y$ lo interesante del caso es que durante dicho año la productividad del trabajo, que había estado aumentando desde 1880 , disminuyó de forma bastante acusada, mientras que el coste medio del trabajo por máquina producida aumentó ${ }^{39}$.

En suma, las conclusiones que pueden deducirse de nuestro análisis son las siguientes:

- El origen de la mecanización de la fundición fue extractivo.

- Lo relevante no fue tanto la maquinaria introducida como el cambio del sistema de control impuesto ${ }^{40}$.

- El problema fundamental para la empresa no era la mayor eficiencia inmediata, tanto física como extractiva, sino la consecución de un sistema de control que le diera garantías de continuidad.

\section{La introducción de maquinaria especializada en la década de 1890}

La victoria obtenida por la compañía en 1886 estableció un sistema de control más eficiente en la fundición y restableció la eficiencia del sistema

39 Los datos sobre producción, productividad, empleo, costes y beneficios pueden hallarse en Toharia (1979), cap. 2.

to Es decir, el cambio tecnológico tue en realidad un cambio "no incorporado", similar al de 1880 . 
implantado en 1880 en el resto de la factoría. Ello, unido a la fuerte mecanización experimentada por la empresa durante los años siguientes, explica, sin duda, el hecho de que la productividad del trabajo se triplicara con creces entre 1886 y 1892 .

El tercer cambio técnico objeto de nuestra atención en este artículo es precisamente la mecanización que acabamos de mencionar. La mayor parte de las máquinas introducidas eran máquinas especializadas, fabricadas expresamente para la producción de maquinaria agrícola, y su diseño y construcción fueron realizados en la propia factoría McCormick. Los departamentos que se vieron más afectados fueron la carpintería y la herrería, que eran los que empleaban una proporción mayor de trabajadores cualificados.

Así, pues, parece que nos hallamos ante otro caso más de sustitución de trabajadores cualificados por maquinaria más eficiente y productiva. Sin embargo, lo interesante del caso, y lo que hace que lo presentemos como evidencia del enfoque teórico adoptado en este artículo, es que los directivos de la compañía McCormick no percibían la introducción de esas máquinas desde el punto de vista de la eficiencia «física», sino desde el punto de vista de la extracción de trabajo. La principal prueba de ello la constituye la carta que H. B. Utley, superintendente de la fábrica, dirigió a Nettie F. McCormick, viuda de Cyrus y principal accionista de la compañía, explicando los motivos de estos cambios:

«Los gastos en mejora permanente han sido muy pequeños y la principal partida ha correspondido a herramientas y máquinas especiales diseñadas y construidas por nosotros mismos. Consideramos que este gasto constituye una inversión rentable. El fabricar aparejos especiales nos permite utilizar menos trabajo cualificado, ya que se fabrican herramientas que hagan el trabajo de los mecánicos cualificados. En este sentido, cada año que pasa somos más independientes del trabajo. De hecho, la cuestión de las perturbaciones laborales es el menos importante de nuestros problemas..." ${ }^{41}$.

Esta carta, escrita en 1894, en una época de crisis de la economía americana, refleja muy nítidamente las motivaciones subyacentes a la mecanización. En una época de producción y beneficios crecientes, probablemente no habría sido necesario dar una explicación de los gastos en maquinaria. Y lo interesante es que, incluso en un período de crisis, Mr. Utley podría haber explicado la introducción de las máquinas aduciendo su mayor productividad

4 Esta carta procede de los Cyrus H. McCormick Papers, serie 1, caja 111, McCormick Collection. 
física y, por tanto, su menor incidencia en los çostes de producción. Sin embargo, su explicación fue muy diferente, ya que ligaba la independencia con respecto a los trabajadores cualificados con la inexistencia de problemas laborales. Las "perturbaciones laborales» a que se refería $\mathrm{Mr}$. Utley pueden interpretarse como perturbaciones de la extracción de trabajo.

En suma, la introducción de maquinaria especializada en la década de 1890 es una clara ilustración de cambio técnico cuya motivación era «extractiva», aunque un análisis superficial a posteriori podría habernos hecho creer que se trataba meramente de cambios que pretendían alterar el proceso técnicoeconómico y que tuvieron unas consecuencias extractivas inevitables e indeseadas. Nuestro análisis sugiere, por tanto, que los análisis superficiales y a posteriori son deficientes. El problema es, obviamente, que los análisis de las motivaciones no son fáciles de documentar. De ahí el posible relieve de un análisis histórico como el que hemos realizado en este artículo que permita descender al detalle y que se base en documentos poco relevantes para el funcionamiento actual de las empresas y, por tanto, accesibles al investigador.

\section{Conclusión}

Los tres cambios técnicos analizados en este artículo constituyen una sólida evidencia de que el marco teórico propuesto en el apartado 2, y en concreto la posibilidad de que los cambios técnicos vengan motivados por razones «extractivas», tiene relevancia empírica. Como ya quedó apuntado en la introducción, ello implica que la idea del determinismo tecnológico, según la cual el progreso tecnológico es un fenómeno que se impone en base a su mayor eficiencia productiva, es un mito que debe ser rechazado. Los cambios tecnológicos, tanto los que llevan incorporados nuevas máquinas y equipos como los que no los llevan, como son los cambios organizativos, son introducidos, algunas veces, porque son más eficientes desde el punto de vista de la productividad física $y$, otras, porque permiten a la empresa una conversión de su fuerza de trabajo en trabajo efectivo más eficiente. No pretendemos ni nos importa cuantificar las proporciones correspondientes a cada uno de los dos tipos, aparte de que no siempre son separables. Lo que nos interesa es destacar que los motivos extractivos, independiente o conjuntamente con los físicos, son relevantes en las sociedades capitalistas, en las que las relaciones laborales constituyen un elemento importante, cuando no definitorio. 


\section{BIBLIOGRAFIA}

Ashworth, John H. (1915): The Helper and American Trade Unions, Baltimore, John Hopkins University Press.

Braverman, Harry (1974), Labor and Monopoly Capital, Nueva York, Monthly Review Press.

BREcher, Jeremy (1972), Strike!, San Francisco, Straight Arrow Books.

Brody, David (1960): Steelworkers in America, Cambridge, Mass., Harvard University Press.

David, Paul A. (1975): "The Mechanization of Reaping in the Antebellum Midwest", en su Technical Choice, Innovation and Economic Growth, Cambridge, Cambridge University Press. pp. 195-232.

Duborsky, Melvyn (1975): Industrialism and the American Worker, 1865-1920, Arlington Heights, Ill, AHM Publishing Co.

EDwards, Richard C. (1979): Contested Terrain, Nueva York, Basic Books.

GintIs, Herbert, y Bowles, Samuel (1981): "Structure and Practice in the Labor Theory of Value", The Review of Radical Political Economics, vol. 12, núm, 4, invierno, pp. 1-26.

Gordon, David M.; EDwards, Richard C., y REIch, Michael (1982): Segmented Work, Divided Workers, Cambridge, Cambrigde University Press.

Henderson, Jeff, y CoHen, Robin (1979): "Capital and the Work Ethic", Monthly Review, vol. 31, núm. 6, noviembre, pp. 11-26.

Hounshell, David A. (1978): From the American System to Mass Production: The Development of Manufacturing Technology in the United States, 1850-1920, tesis doctoral inédita, Departamento de Historia, University of Delaware.

Hutchinson, T. W. (1930): Cyrus Hall McCormick, Seed-Time, 1809-1856, Nueva York, D. Appleton-Century Co.

- (1935): Cyrus Hall McCormick, Harvest, 1856-1884, Nueva York, D. AppletonCentury Co.

JoNEs, Lewis R. (1977): "The Mechanization of Reaping and Mowing in American Agriculture, 1833-1870: Comment", Journal of Economic History, vol. 37, número 2, junio, pp. 451-455.

Marglin, Stephen A. (1974): "What Do Bosses Do? The Origins and Functions of Hierarchy in Capitalist Production", The Review of Radical Political Economics, vol. 6, núm. 2 , verano, pp. 33-60.

Nelson, Richard R. (1981): "Research on Productivity Growth and Productivity Differences: Dead Ends and New Departures", Journal of Economic Literature, septiembre, pp. 1029-1064.

Olmstead, Alan L. (1975): "The Mechanization of Reaping and Mowing in American Agriculture, 1833-1870", Journal of Economic History, vol. 35, núm. 2, junio, pp. 327-352.

OzAnNe, Robert (1967): A Century of Labor-Management Relations at McCormick and International Harvester, Madison, Wis., Wisconsin University Press.

- (1968): Wages in Practice and Theory, McCormick and International Harvester, 1860-1960, Madison, Wis., Wisconsin University Press.

PARKER, William, y KLEIN, Judith (1966): "Productivity Growth in Grain production in the United States, 1840-1860 and 1900-1910", en Conference on Research in Income and Wealth, Output, Employment and Productivity in the United States after 1800, Nueva York, Columbia University Press, pp. 523-580.

Reich, Michael, y Devine, James (1981): "The Microeconomics of Conflict and Hierarchy in Capitalist Production", The Review of Radical Political Economics, vol. 12, núm. 4, invierno, pp. 27-45.

RoE, J. W., y LyTLE, C. W. (1935): Factory Equipment, Scranton, PA, International Textbook Company. 
Rosenberc, Nathan (1969): "The Direction of Technological Change: Inducement Mechanisms and Focusing Devices", Economic Development and Cultural Change, vol. 18, núm. 1, octubre, pp. 3-24.

Samuelson, Paul A. (1957): "Wages and Interest: A Modern Dissection of Marxian Economic Models", American Economic Review, vol. 47, núm. 5, diciembre, páginas 884-912.

Smrt, Merritt Roe (1977): Harpers Ferry Armory and the New Technology, Ithaca, N. Y., Cornell University Press.

Stockton, Frank T. (1922): The International Molders Union of America, Baltimore, Johns Hopkins University Press.

STONE, Katherine (1974): "The Origins of Job Structures in the Steel Industry", The Review of Radical Political Economics, vol. 6, núm. 2, verano, pp. 61-97.

TAYLOR, Frederick Winslow (1895): "A Piece-rate System, Being a Step Toward Partial Solution of the Labor Problem", A.S.M.E. Transactions, vol. XVI, páginas 856-889.

Temin, Peter (1966): "Labor Scarcity and the Problem of American Industrial Efficiency in the 1850s", Journal of Economic History, vol. 26, septiembre, páginas $361-379$.

- (1971): "Labor Scarcity in America", Journal of Interdisciplinary History, volumen 1, pp. 251-264.

ToHARIA, Luis (1979): The Division of Labor and the Historical Development of the Internal Labor Market: A Case-study of the McCormick Works of Chicago. 1848-1902, tesis doctoral inédita, Departamento de Economía, Massachusetts Institute of Technology.

U. S. EMPloYment SERvice (1938): Job Descriptions for Job Foundries, Washington, D.C., U.S. Department of Labor.

Vegara, Josep M." (1981): "Fuerza de trabajo y trabajo", Revista Mensual/Monthly Review, vol. 4, núm. 5, febrero, pp. 7-14. 